\title{
Kathrin Vogler (Die Linke), MdB
}

\author{
Mitglied im Gesundheitsausschuss des Deutschen Bundestags
}

Für ihre Arbeit bekommt die CDU/CSU-SPD-Koalition von mir ein Fleißkärtchen, aber keine guten Noten. Denn sie hat die Umverteilung von unten nach oben fortgesetzt oder sogar noch verschärft, etwa durch die Festschreibung der Arbeitgeberbeiträge in der gesetzlichen Krankenversicherung, durch die Finanzierung gesamtgesellschaftlicher Aufgaben wie Krankenhausstruktur, gesundheitliche Aufklärung oder Innovationsfonds durch die gesetzlich Versicherten und durch die massiv angestiegenen Ausgaben für neue, nicht immer innovative Medikamente. Nun greift sie auch noch in den Gesundheitsfonds, damit die Beiträge nicht schon im Wahljahr ansteigen, sondern erst danach. Das ist ein Armutszeugnis.

Die Selbstverwaltung durch Körperschaften öffentlichen Rechts ist eine international recht einmalige Konstruktion. Der Staat, der für die Sicherstellung der gesundheitlichen Versorgung seiner Bürgerinnen und Bürger verantwortlich ist, überträgt dieser Selbstverwaltung wichtige Entscheidungen über die Verwendung von Mitteln, die gesetzlich Versicherte mit ihren Pflicht- beiträgen aufbringen. Wenn diese Strukturen ihren Versorgungsauftrag nicht angemessen wahrnehmen, wenn sie - wie bei der KBV - obskures Finanzgebaren entwickeln oder - wie beim „Upcoding“ der Krankenkassen - die Solidargemeinschaft betrügen, dann muss der Staat im Sinne der Allgemeinheit eingreifen. Wer sich anschauen will, was die komplette Privatisierung und Wettbewerbsorientierung im Gesundheitswesen für Auswirkungen hat, muss nur in die USA schauen: Sozial ungleicher Zugang und explodierende Kosten sind das Ergebnis. Das wollen wir hier nicht.

Wir werden uns im Wahljahr und erst recht in der nächsten Legislaturperiode für eine solidarische Gesundheits- und Pflegeversicherung einsetzen. Unser Ziel: eine umfassende Versorgung für alle zu bezahlbaren, sozial gerechten Beiträgen auf alle Einkommensarten und paritätischer Finanzierung. Auch eine gesetzliche Mindestpersonalbemessung in der Pflege und die Versorgung mit sicheren, wirksamen und bezahlbaren Arzneimitteln stehen oben auf der Agenda.

\section{Maria Klein-Schmeink (Bündnis90/Die Grünen), MdB}

\author{
Gesundheitspolitische Sprecherin der Fraktion Bündnis 90/Die Grünen im Bundestag
}

Die Koalition hat wirklich sehr viele Gesetze durch das Parlament gebracht. Aber Masse ist nicht unbedingt gleichbedeutend mit Klasse. Da wurden zum Teil sehr kleinteilig die Regelungen zur Versorgung flexibilisiert, viele Aufgaben an die Selbstverwaltung delegiert, wo man am Ende nicht weiß, was wirklich herauskommen wird. Bei den großen Strukturproblemen in unserem Gesundheitssystem hat die Koalition sich dagegen selbst blockiert. Das betrifft insbesondere das Finanzierungssystem. Fast jedem ist doch klar: Wir müssen unser Gesundheitssystem stabiler und gerechter finanzieren.

Zudem wurden zwei sehr wesentliche und auch kostenträchtige Reformen in die nächsten Wahlperioden vertagt: die verbindliche Personalbemessung in der stationären Krankenpflege und in der Altenpflege. Das ist angesichts der äußerst fordernden Arbeitsbedingungen und des schon schwerwiegenden Fachkräftemangels in diesen Bereichen ein unverantwortliches Versäumnis.

Auch der Arztberuf steht vor einem Wandel. In Zukunft kommt es stärker noch als bisher auf teamorientierte Zusammenarbeit mit anderen Gesundheitsberufen an. Deswegen glaube ich, dass hier ein Schwerpunkt in der Reform des Medizinstudiums gesetzt werden sollte. Ein weiterer wichtiger As-

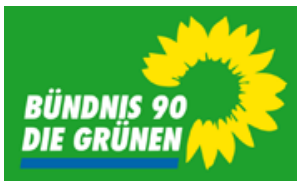
pekt ist die Digitalisierung des Gesund-

heitswesens. Das wird unser Gesundheitswesen erheblich verändern, auch die Ärztinnen und Ärzte müssen sich darauf einstellen. Wir müssen also in der Ausbildung auch Augenmerk auf die Digitalkompetenz der künftigen Ärztinnen und Ärzte legen.

Auch das Verhältnis zwischen Patienten und Ärzten verändert sich. Patientinnen und Patienten informieren sich selbst immer stärker über geeignete Therapien. In der Ausbildung muss die Kommunikation auf Augenhöhe und eine partizipative Entscheidungsfindung trainiert werden.

Im Bereich E-Health ist die Bundesregierung gesetzgeberisch nicht über den Bereich der Telematik hinausgekommen, und selbst da muss noch einiges passieren, damit der Rollout der elektronischen Gesundheitskarte nicht ewig ein Rohrkrepierer bleibt. Die Telemedizin und der sich rasant entwickelnde Markt der Gesundheits-Apps kommen im E-Health-Gesetz erst gar nicht vor. Hier muss die Bundesregierung schleunigst nachbessern und für verbindliche, nachvollziehbare Qualitäts- und Sicherheitsstandards sorgen. 